\title{
SEMIGROUP ACTION FOR HUTCHITSON OPERATOR OF ITERATED FUNCTION SYSTEM
}

\author{
1.Esmaeel Rezaali, 2. Mohsen Saleh \\ 1. Department of Mathematics, Ferdowsi University of Mashhad, Mashhad, Iran \\ esmaeel.rezaali@stu.um.ac.ir \\ esmaeel.rezaali@gmail.com \\ 2. Department of Mathematics, University of Neyshabur, Neyshabur, Iran \\ saleh@neyshabur.ac.ir
}

\begin{abstract}
In this article, we are trying to see the Iterated function system or more generally a skew product as formation a semigroup action system. We will try to implement more semigroup action properties on itself. For this purpose, we introduce a semigroup action corresponding Iterated function system and explain more properties of itself then with considering Hutchitson operator on Iterated function system, we try consider the corresponding semigroup action for Iterated function system and achive more its properties.
\end{abstract}

Keywords: Iterated function systems; Minimality; Transitivity; Hutchitson operator; Semigroup action.

Mathematics; Dynamical Systems.

\section{Mathematics Subject Classification; 37B05, 37C85, 54H20.}

\section{Introduction}

Iterated function systems is a fast developing topic which has been studied extensively during the last decade. An iterated function system is a finite set of contraction mappings on a complete metric space. One major reason for this recent activity was the introduction of the fractal concept in the seventies, which led to question on how to create new fractals. An important method is to use iterated function systems (IFS), (see Barnsley and Demko [1985]).

Problems concerning more effective image building naturally led to the introduction of iterated function systems with probabilities, i.e. iterated function systems controlled by a sequence of independent, identically distributed random variables.

Barnsley, Elton and Hardin (1989) generalized this model. They considered IFS controlled by a Markov chain and called them recurrent IFS, (e.g. [1, 3, 6, 12]).

The aim of present paper is to discuss the behavior of dynamical systems in semigroup actions and reach to relationship of concepts, [6]. We study iterated function systems with Hutchitson operator and find main results.

The present paper is organized as follows: In section 2, we recall some standard defnitions about iterated function systems. In section 3 , we study equicontinuity, transitivity and minimality of the dynamical system $\left(\mathbf{Z}_{+}, \sum_{+}^{n} \times M\right)$ and show that if $\sum_{+}^{n} \times M$ is polish space due to densely point transitivity of $\left(\mathbf{Z}_{+}, \Sigma_{+}^{n} \times M\right)$.

In section 4, we study iterated function systems with Hutchitson operator and show that if $M$ is polish space due to densely set transitivity of every topological transitive system.

\section{PRELIMINARIES}

Let $M$ be a compact metric space and $\sum_{+}^{n}$ be symbol space, We define the dynamical systems as

skew product $\mathrm{F}$ with $\left(\sum_{+}^{n} \times M, \mathrm{~F}\right)$ that

$$
\begin{gathered}
\mathrm{F}: \sum_{+}^{n} \times M \rightarrow \sum_{+}^{n} \times M \\
\mathrm{~F}(\omega, p)=\left(\sigma \omega, f_{\omega}(p)\right), \omega \in \sum_{+}^{n}, p \in M
\end{gathered}
$$

In particular skew product is composition of semi-action of functions on generators $\left\{f_{1}, f_{2}, \ldots, f_{s}\right\}$.

We centralize on fiber $M$, we will be faced with the concept of Iterated function systems shortly IFS.

The iterated function system of these maps, denoted by $\left(M: f_{1}, f_{2}, \ldots, f_{s}\right)$ or shortly 
$\mathrm{IFS}(\mathrm{F})$, is the set of all finite forward composition of these maps.

Let $\mathrm{F}: \sum_{+}^{n} \times M \rightarrow \sum_{+}^{n} \times M$ be a skew product with generators $\left\{f_{1}, f_{2}, \ldots, f_{s}\right\}$, we define following statements. We have

$$
f_{\omega}^{n}(x)=f_{\sigma^{n-1} \omega} \circ \ldots \circ f_{\sigma \omega} \circ f_{\omega}(x)
$$

thus the orbit of $\mathrm{F}$ with respect to $\omega \in \sum_{+}^{n}$ at $x \in M$ is the set

$$
O_{\omega}(x)=\left\{x, f_{\omega}(x), f_{\omega}^{2}(x), \ldots, f_{\omega}^{n}(x), \ldots\right\}
$$

and the total orbit of $\mathrm{F}$ at $x \in M$ is the set

$$
O_{\text {total }}(x)=\left\{f_{\omega}^{n}(x): n \in \square_{0} \text { and } \omega \in \sum_{+}^{n}\right\}
$$

For skew product $F,\left(\omega_{0}, p_{0}\right)$ is periodic of period $n$, if

$$
f^{n}\left(\omega_{0}, p_{0}\right)=\left(\omega_{0}, p_{0}\right)
$$

i.e. $\sigma^{n}\left(\omega_{0}\right)=\omega_{0}$ and $f_{\omega}^{n}\left(p_{0}\right)=p_{0}$.

For every periodic sequence $\omega_{0}=\omega\left(\sigma^{n}\left(\omega_{0}\right)=\omega_{0}\right)$ of period $n$, we say that $p_{0}$ is periodic point of IFS $(\mathrm{F})$ if $f_{\omega_{0}}^{n}\left(p_{0}\right)=p_{0}$.

\section{Iterated function systems as semigroup action}

We know that $\mathbf{Z}_{+}=\{0,1,2, \ldots\}$ with operator "+" is semigroup. Let $\sum_{+}^{n}$ is the set of all one side sequence of $\{1,2, \ldots, n\}$. A dynamical system in the present article is a triple $\left(\mathbf{Z}_{+}, \sum_{+}^{n} \times M, \pi\right)$ where

$$
\pi: \mathbf{Z}_{+} \times\left(\sum_{+}^{n} \times M\right) \rightarrow \sum_{+}^{n} \times M
$$

such that

$$
(n, \omega(p)) \rightarrow\left(\sigma^{n}(\omega), f_{\omega}^{n}(p)\right)
$$

where $M$ is a manifold. For $U \subset \sum_{+}^{n}$ we have

$$
-n U=\left\{(\omega, p) \in \sum_{+}^{n} \times M \quad:\left(\sigma^{n}(\omega), f_{\omega}^{n}(p)\right) \in U\right\}
$$

We have

$$
\pi(n, U)=\left\{\left(\sigma^{n}(\omega), f_{\omega}^{n}(p)\right):(\omega, p) \in U\right\}
$$

Definition 3.1. Let $S$ be a topological semigroup.

(1) We say that $S$ is a $F$-semigroup if for every $s_{0} \in S$ the subset $S \backslash S s_{0}$ that $S s_{0}=\left\{s \in S: s \notin S s_{0}\right\}$ is finite.

Since $\mathbf{Z}_{+} \backslash \mathbf{Z}_{+}+n_{0}=\left\{0,1, \ldots, n_{0}-1\right\}$ is a finite set, then $\left(\mathbf{Z}_{+},+\right)$is a $F$ - semigroup. 
(2) We say that $S$ is a $C$-semigroup if $S \backslash S s_{0}$ is relatively compact (that its closure is compact) in $S$.

Note that every $F$-semigroup is $C$-semigroup, so $\left(\mathbf{Z}_{+},+\right)$is a $C$-semigroup.

Definition 3.2. Let $\left(\mathbf{Z}_{+}, \sum_{+}^{n} \times M\right)$ be a dynamical system where $\left(\sum_{+}^{n} \times M, d\right)$ is a metric space. Where $d=d_{\sigma} \times d_{M}$ such that $d_{\sigma}$ is metric on symbol dynamic and $d_{M}$ is a metric on $M$.

(1) A subset $A$ of $\mathbf{Z}_{+}$act equicontinuity at $\left(\omega_{0}, p_{0}\right) \in \Sigma_{+}^{n} \times M$ if for every $\omega$, for every $\varepsilon>0$ there exists $\delta>0$ such that $d\left(\left(\omega_{0}, p_{0}\right),(\omega, p)\right)<\delta$ implies

(9)

$$
d\left(\left(\sigma^{n}\left(\omega_{0}\right), f_{\omega_{0}}^{n}\left(p_{0}\right)\right),\left(\sigma^{n}(\omega), f_{\omega}^{n}(p)\right)\right)<\varepsilon
$$

for every $n \in A$.

(2) A subset $A$ of $\mathbf{Z}_{+}$act fiber equicontinuity at $p_{0} \in M$ with respect $\omega_{0}$ if for every $\varepsilon>0$ there exists $\delta>0$ such that $d_{M}\left(p_{0}, p\right)<\delta$ implies that

$$
d\left(f_{\omega_{0}}^{n}\left(p_{0}\right), f_{\omega_{0}}^{n}(p)\right)<\varepsilon
$$

for every $n \in \mathbf{Z}_{+}$.

(3) A point $p_{0} \in M$ is called an orbital equicontinuity point with respect to $\omega_{0}$ ( $\left.p_{0} \in E q_{\omega_{0}}(M)\right)$ if $\mathbf{Z}_{+}$act orbital equicontinuity at $p_{0}$ with respect to $\omega_{0}$.

The fiber $\omega_{0}$ is equicontinuous fiber if $E q_{\omega_{0}}(M)=M$.

(4) The orbital $\omega_{0}$ is called almost orbital equicontinuous if the subset $E q_{\omega_{0}}(M)$ of orbital equicontinuity points is dense subset of $M$.

Definition 3.3. The dynamical system $\left(\mathbf{Z}_{+}, \Sigma_{+}^{n} \times M\right)$ is called

(1) topological transitive if for every $(U, V)$ of nonempty open sets $U, V$ in $\sum_{+}^{n} \times M$ there exists $n \in \mathbf{Z}_{+}$such that $\pi(n, U) \cap V=\varnothing$.

(2) Point transitive if there exists a point $\left(\omega_{0}, p_{0}\right)$ with dense orbit

$$
\mathbf{Z}_{+}\left(\omega_{0}, p_{0}\right)=\left\{\left(\left(\sigma^{n}\left(\omega_{0}\right), f_{\omega_{0}}^{n}\left(p_{0}\right)\right): n \in \mathbf{Z}_{+}\right\}\right.
$$

is dense in $\sum_{+}^{n} \times M$. Such a point $\left(\omega_{0}, p_{0}\right)$ is called transitive point,

$$
\left(\omega_{0}, p_{0}\right) \in \operatorname{tran}\left(\sum_{+}^{n} \times M\right)
$$

(3) Densely point transitive if there exists a dense set $Y \subset \Sigma_{+}^{n} \times M$ of transitive points.

(4) Orbital topological transitive with respect $\omega_{0}$, if for every nonempty open sets $U, V$ in $M$, there exists $n \in \mathbf{Z}_{+}$such that $f_{\omega_{0}}^{n}(U) \bigcap V \neq \varnothing$.

(5) Orbital point transitive with respect to $\omega_{0}$, if there exists a point $p_{0}$ with dense orbit 


$$
\mathbf{Z}_{+_{\omega_{0}}}=\left\{f_{\omega_{0}}^{n}(p): n \in \mathbf{Z}_{+}, p \in M\right\} \text { in } M
$$

(6) Orbital densely point transitive if there exists a dense set $Y \subset M$ of orbital transitive points.

Lemma 3.4. Consider $\left(\mathbf{Z}_{+}, \sum_{+}^{n} \times M\right)$ as orbital topological transitive with respect to $\omega_{0}$, if $p \in E q_{\omega_{0}}, O_{\omega_{0}}(p)$ is dense in $M$.

Proof. We need to show that for every $x \in M$ and $\varepsilon>0$ there exists $n \in \mathbf{Z}_{+}$such that

$$
d_{M}\left(f_{\omega_{0}}^{n}(p), x\right)<\varepsilon
$$

Consider $\varepsilon^{\prime}=\frac{\varepsilon}{2}$, by definition of equicontinuity, there exists $\delta>0$ such that if

$d_{M}(p, q)<\delta, q \in M$, then for every $n \in \mathbf{Z}_{+}$,

$$
d_{M}\left(f_{\omega_{0}}^{n}(p), f_{\omega_{0}}^{n}(q)\right)<\varepsilon^{\prime}
$$

Now consider $U=B_{\delta}(p), V=B_{\varepsilon^{\prime}}(x)$, by definition of orbital topological transitive with respect to $\omega_{0}$, there exists $n_{0} \in \mathbf{Z}_{+}$such that $f_{\omega_{0}}^{n_{0}}(U) \cap V \neq \varnothing$. Therefore there exists $y \in U$ such that

$$
d_{M}\left(f_{\omega_{0}}^{n_{0}}(y), x\right)<\varepsilon^{\prime}
$$

Now we have

$$
d_{M}\left(f_{\omega_{0}}^{n_{0}}(p), x\right) \leq d_{M}\left(x, f_{\omega_{0}}^{n_{0}}(y)\right)+d_{M}\left(f_{\omega_{0}}^{n_{0}}(y), f_{\omega_{0}}^{n_{0}}(p)\right)<\varepsilon^{\prime}+\varepsilon^{\prime}=\varepsilon
$$

Corollary 3.5. Let $\left(\mathbf{Z}_{+}, \sum_{+}^{n} \times M\right)$ is orbital topological transitive with respect to $\omega_{0}$. If $E q_{\omega_{0}} \neq \varnothing$, then $\left(\mathbf{Z}_{+}, \sum_{+}^{n} \times M\right)$ is orbital point transitive.

Proof. According to lemma 3.4 is trivial.

Lemma 3.6. Consider dynamical system $\left(\mathbf{Z}_{+}, \sum_{+}^{n} \times M\right)$. Let $A \subseteq \mathbf{Z}_{+}$be a finite subset,

Then $A$ act equicontinuously on $\left(\sum_{+}^{n} \times M, d\right)$.

Proof. This lemma holds trivial, because continuity in finite set implies equicontinuity.

In this article we consider $M$ as perfect Reimanian manifold so $\sum_{+}^{n} \times M$ is a space without isolated points.

Densely point transitive implies point transitive and point transitive implies topological transitive.

Note that $\left(\sum_{+}^{n}, d_{\delta}\right)$ is a polish space but $\left(M, d_{M}\right)$ (and so $\left.\left(\sum_{+}^{n} \times M, d\right)\right)$ can be a polish space or not.

Proposition 3.7. If $\sum_{+}^{n} \times M$ is a polish space, then every topological transitive system

$\left(\mathbf{Z}_{+}, \sum_{+}^{n} \times M\right)$ is densely point transitive (and hence also point transitive).

Proof. If $\left(\mathbf{Z}_{+}, \sum_{+}^{n} \times M\right)$ is topological transitive, then $-\mathbf{Z}_{+} U$ is a dense subset of $\Sigma_{+}^{n} \times M$, for every 
open set $U$. We know that $\sum_{+}^{n} \times M$ is polish, then there exists a countable open

base $B$ of the given topology. By the Baire space theorem $\bigcap\left\{-\mathbf{Z}_{+} U: U \in B\right\}$ is dense in $\sum_{+}^{n} \times M$ and every point of this set is a transitive point of the dynamical system $\sum_{+}^{n} \times M$.

Definition 3.8. (1) $\sum_{+}^{n} \times M$ is called minimal, if

$$
\overline{\mathbf{Z}_{+}(\omega, p)}=\sum_{+}^{n} \times M
$$

In other words, all points of $\sum_{+}^{n} \times M$ are transitive points.

(2) A point $(\omega, p)$ is called minimal if the subsystem $\overline{\mathbf{Z}_{+}(\omega, p)}$ is minimal.

(3) A point $(\omega, p)$ is called almost periodic if the subsystem $\overline{\mathbf{Z}_{+}(\omega, p)}$ is minimal and compact.

(4) If the set of almost periodic points is dense in $\sum_{+}^{n} \times M$, we say that $\left(\mathbf{Z}_{+}, \Sigma_{+}^{n} \times M\right)$ satisfies the Bronstein condition. If in addition the system $\left(\mathbf{Z}_{+}, \Sigma_{+}^{n} \times M\right)$ is topological transitive, we say that it is an $M$-system.

(5) Let $\left(\sum_{+}^{n} \times M, f\right)$ be a classical discrete dynamical system, as usual, a point $(\omega, p) \in \Sigma_{+}^{n} \times M$ is periodic, if there exists a natural $n \in \mathbf{Z}_{+}$such that $\sigma^{n}(\omega)=\omega$ and $f_{\omega}^{n}(p)=p$.

If $\sum_{+}^{n} \times M$ is a topological transitive and the set of periodic points is dense in $\Sigma_{+}^{n} \times M$, then we say that it is a $P$-system.

If $\sum_{+}^{n} \times M$ is compact then a point in $\sum_{+}^{n} \times M$ is minimal iff it is almost periodic. Therefore it is also abvious that every $P$-system is an $M$-system.

For a system $\left(\mathbf{Z}_{+}, \sum_{+}^{n} \times M\right)$ and a subset $B \subset \sum_{+}^{n} \times M$, we use the following notation

$$
\square((\omega, p), B)=\left\{n \in \mathbf{Z}_{+}:\left(\sigma^{n} \omega, f_{\omega}^{n}(p)\right) \in B\right\}
$$

Definition 3.9. A subset $P \subset \mathbf{Z}_{+}$is (left) syndetic, if there exists a finite set $F \subset \mathbf{Z}_{+}$such that $-F P=\mathbf{Z}_{+}$.

Lemma 3.10. Let $\sum_{+}^{n} \times M$ be a (not necessarily compact) $\mathbf{Z}_{+}$-dynamical system and $\left(\omega_{0}, p_{0}\right) \in \sum_{+}^{n} \times M$. consider the following conditions.

(1) $\left(\omega_{0}, p_{0}\right)$ is an almost periodic point.

(2) For every neighborhood $V$ of $\left(\omega_{0}, p_{0}\right) \in \sum_{+}^{n} \times M$ there exists a finite set $F \subset \mathbf{Z}_{+}$such that

$$
-F V \supseteq Y=\overline{\mathbf{Z}_{+}\left(\omega_{0}, p_{0}\right)} .
$$

(3) for every neighborhood $V$ of $\left(\omega_{0}, p_{0}\right) \in \sum_{+}^{n} \times M$ the set $\square\left(\left(\omega_{0}, p_{0}\right), V\right)$ is syndetic. 
(4) $\left(\omega_{0}, p_{0}\right)$ is a minimal point (i.e. the subsystem $\overline{\mathbf{Z}_{+}(\omega, p)}$ is minimal).

Then (1) implies (2) , (2) implies (3) and (3) implies (4).

If $\sum_{+}^{n} \times M$ is compact then all four conditions are equivalent.

Proof (1) $\rightarrow(\mathbf{2})$ : Suppose that $\left(\mathbf{Z}_{+}, Y\right)$ is minimal and compact that $Y=\overline{\mathbf{Z}_{+}(\omega, p)}$, then for every open neighborhood $V$ of $\left(\omega_{0}, p_{0}\right) \in \Sigma_{+}^{n} \times M$, for every $\left(\omega_{1}, p_{1}\right) \in Y$ there exists $n \in \mathbf{Z}_{+}$such that $f_{\omega_{1}}^{n}\left(p_{1}\right) \in V$. Equivalently $f_{\omega_{1}}^{n}\left(p_{1}\right) \in-n V$. Therefore $\bigcup_{n \in \mathbf{Z}_{+}}-n V \supseteq Y$. By compactness of $Y$ we can choose a finite set $F \subseteq \mathbf{Z}_{+}$such that $-F V \supseteq Y=\overline{\mathbf{Z}_{+}\left(\omega_{0}, p_{0}\right)}$.

$(2) \rightarrow(3):$ It suffics to show that

$$
-F \square\left(\left(\omega_{0}, p_{0}\right), V\right)=\mathbf{Z}_{+}
$$

in otherwise there exists $n \in \mathbf{Z}_{+}$such that

$$
n \notin-F \square\left(\left(\omega_{0}, p_{0}\right), V\right)
$$

then

$$
\left(\sigma^{n} \omega_{0}, f_{\omega_{0}}^{n}\left(p_{0}\right)\right) \notin-F V
$$

On the other hand clearly $\left(\sigma^{n} \omega_{0}, f_{\omega_{0}}^{n}\left(p_{0}\right)\right) \in Y$, contrary to our condition that $-F V \supseteq Y$.

(3) $\rightarrow$ (4) : $Y=\overline{\mathbf{Z}_{+}(\omega, p)}$ is nonempty, closed and invariant. It remains to show that if $\left(\omega_{1}, p_{1}\right) \in Y$ then $\left(\omega_{0}, p_{0}\right) \in \overline{\mathbf{Z}_{+}\left(\omega_{1}, p_{1}\right)}$. Assume otherwise, so that $\left(\omega_{0}, p_{0}\right) \notin \overline{\mathbf{Z}_{+}\left(\omega_{1}, p_{1}\right)}$. By the regularity of $\Sigma_{+}^{n} \times M$, we can choose an open neighborhood $V$ of $\left(\omega_{0}, p_{0}\right) \in \Sigma_{+}^{n} \times M$ such that

$$
V \cap \mathbf{Z}_{+}\left(\omega_{1}, p_{1}\right)=\varnothing
$$

By our assumption the set $\square\left(\left(\omega_{0}, p_{0}\right), V\right)$ is syndetic. Therefore there is a finite set $F=\left\{n_{1}, \ldots, n_{k}\right\}$ so that for every $n \in \mathbf{Z}_{+}$some

$$
\left(\sigma^{n_{i}+n} \omega_{0}, f_{\omega_{0}}^{n_{i}+n}\left(p_{0}\right)\right) \in V
$$

that is

$$
\left(\sigma^{n} \omega_{0}, f_{\omega_{0}}^{n}\left(p_{0}\right)\right) \in-F V=\bigcup_{i=1}^{k}-n_{i} V \text { for every } n \in \mathbf{Z}_{+}
$$

Hence

$$
\mathbf{Z}_{+}\left(\omega_{0}, p_{0}\right) \subseteq \bigcup_{i=1}^{k}-n_{i} V
$$

then 


$$
\left(\omega_{1}, p_{1}\right) \in \overline{\mathbf{Z}_{+}\left(\omega_{0}, p_{0}\right)} \subset \overline{\bigcup_{i=1}^{k}-n_{i} V}=\bigcup_{i=1}^{k} \overline{-n_{i} V} \subset \bigcup_{i=1}^{k}-n_{i} \bar{V}
$$

contrary to our assumption.

If $\sum_{+}^{n} \times M$ is compact then by definition it follow that (4) implies (1).

\section{Iterated function systems with Hutchitson operator}

We consider $M$ as compact manifold and IFS $\left\{M, f_{1}, \ldots, f_{k}\right\}$. We can use Hutchitson operator for constructing semigroup. We have set

$$
\varphi=\{A \subseteq M: A \text { is compact }\}
$$

and

$$
\begin{gathered}
\mathrm{H}: \boldsymbol{\varphi} \rightarrow \boldsymbol{\varphi} \\
\mathrm{H}(A)=f_{1}(A) \cup \ldots \cup f_{k}(A)
\end{gathered}
$$

Note that for every open set $U$ we have

$$
\mathrm{H}(U)=f_{1}(U) \cup \ldots \cup f_{k}(U)
$$

Thus $\mathrm{H}$ under function composition is semigroup and isomorphic to $\mathbf{Z}_{+}$. Therefore we explain more definitions for $\mathrm{H}$.

1) Compact set $A \subset M$ is equicontinuous if for every $\varepsilon>0$ there exists $\delta>0$ such that for every compact set $B \subset M$,

then

$$
d_{H}(A, B)<\delta
$$

$$
d_{H}\left(\mathrm{H}^{n}(A), \mathrm{H}^{n}(B)\right)<\mathcal{E} \text {, for every } n \in \mathbf{Z}_{+} .
$$

Particularly if $A=\{a\}$ is equicontinuous means, for every $\varepsilon>0$ there exists $\delta>0$ such that for every $b \in M, d_{M}(a, b)<\delta$ then for every $\omega \in \Sigma_{+}^{n}, n \in \mathbf{Z}_{+}$,

$$
d_{M}\left(f_{\omega}^{n}(a), f_{\omega}^{n}(b)\right)<\varepsilon
$$

We say that $M$ is equicontinuous if for every compact set $A \subset M, A$ is equicontinuous set.

2) Operator $\mathrm{H}$ is almost equicontinuous if $M$ be a equicontinuous set.

3) Operator $\mathrm{H}$ is topological transitive if for every nonempty open sets $U, V$ there exists

compact set $A \subset U, n \in \square$ that

$$
\mathrm{H}^{n}(A) \bigcap V \neq \varnothing
$$

or operator $\mathrm{H}$ is topological transitive if for every nonempty open sets $U, V$, there exists $n \in \square$ that $\mathrm{H}^{n}(A) \bigcap V \neq \varnothing$. 
4) $M$ is set transitive if there exists compact set $A \subseteq M$ such that

$$
\overline{O_{H}(A)}=M
$$

and $A$ is set transitive if $\overline{O_{H}(A)}=M$.

Let $D$ be a collection of compact sets $B_{\alpha}$ such that for every compact set $A \subseteq M$ and for every $\varepsilon>0$ there exists compact set $B_{\alpha} \in D$ such that $d_{H}\left(A, B_{\alpha}\right)<\varepsilon$.

$D$ is dense in $\varphi$ if $D=\varphi$.

5) $M$ is densely transitive if for every $\varepsilon>0$ and compact set $A \subseteq M$, there exists $B \in D, d(A, B)<\varepsilon$ and $B$ is transitive set.

6) $M$ is polish space means that a separable metric space which is homomorphic to a complete metric space.

Lemma 4.1. Consider $M$ as compact manifold. Let $A \subseteq \mathbf{Z}_{+}$be a finite set. Then $A$ act equicontinuously on $\varphi$.

Proof. According to continuity of operator $\mathrm{H}$ is trivial.

For every compact set $B \subset M$, for every $\varepsilon>0$, there exists $\delta>0$ such that for every $B^{\prime} \in \varphi, d_{H}\left(B, B^{\prime}\right)<\delta$ then

$$
d_{H}\left(\mathrm{H}^{n}(B), \mathrm{H}^{n}\left(B^{\prime}\right)\right)<\varepsilon \text {, for every } n \in A \text {. }
$$

Let $A=\left\{n_{1}, \ldots, n_{k}\right\}$ and $B \subseteq M, B$ is compact subset. We know that $f_{1}, \ldots, f_{l}$ (generator of IFS) is continuous, so for every $\varepsilon>0$ there exists $\delta_{i, 1}$ for $f_{i}$ such that if

$$
d_{H}\left(B, B^{\prime}\right)_{<} \delta_{i, 1}
$$

then

$$
d_{H}\left(f_{i}(B), f_{i}\left(B^{\prime}\right)\right)<\varepsilon
$$

Then if

$$
d_{H}\left(B, B^{\prime}\right)<\min \left\{\delta_{i, 1}\right\}_{i=1}^{l}
$$

then

$$
d_{H}\left(\bigcup_{i=1}^{l} f_{i}(B), \bigcup_{i=1}^{l} f_{i}\left(B^{\prime}\right)\right)<\varepsilon
$$

Therefore $d_{H}\left(B, B^{\prime}\right)<\min \left\{\delta_{i}\right\}_{i=1}^{l}$ then

$$
d_{H}\left(\mathrm{H}(B), \mathrm{H}\left(B^{\prime}\right)\right)<\varepsilon
$$

If $\varepsilon=\min \left\{\delta_{i}\right\}$ then there exists $\delta_{i, 2}$ that if 


$$
d_{H}\left(B, B^{\prime}\right)<\min \left\{\delta_{i, 1}, \delta_{i, 2}\right\}_{i=1}^{l}
$$

therefore

$$
d_{H}\left(\mathrm{H}(B), \mathrm{H}\left(B^{\prime}\right)\right)<\min \left\{\delta_{i}\right\}_{i=1}^{l}
$$

then

$$
d_{H}\left(\mathrm{H}^{2}(B), \mathrm{H}^{2}\left(B^{\prime}\right)\right)<\varepsilon
$$

Under finite repeats, with minimizing initial $\delta$ if

$$
d_{H}\left(B, B^{\prime}\right)<\min \left\{\delta_{i, j}, i=1, \ldots, l, j=1, \ldots, n_{k}\right\},
$$

then

$$
d_{H}\left(\mathrm{H}^{s}(B), \mathrm{H}^{s}\left(B^{\prime}\right)\right)<\varepsilon \text { for every } s=0,1, \ldots, n_{k} .
$$

Because $A \subseteq\left\{0,1, \ldots, n_{k}\right\}$ then every compact set is equicontinuous with respect to $A$ and proof is complete.

Proposition 4.2. If $M$ is polish space, then every toplogical transitive system is densely set transitive.

Proof. For proving we need to show that for every $\varepsilon>0$ and for every compact set $A$, there exists a compact set $B \subset M$ such that $d_{H}(A, B)<\varepsilon, B$ is set transitive. We must suppose that compact set $A, \varepsilon>0$ are arbitrary. We need to show that there exists compact set $B \subset M$, that $d_{H}(A, B)<\varepsilon$, is set transitive. Then we must show that

$$
M=\lim _{n \rightarrow \infty} \overline{\bigcup_{i=n}^{\infty} \mathrm{H}^{n}(B)}
$$

means that for open set $V$ must there exists $\left\{n_{i}\right\} \rightarrow \infty$,

$$
\mathrm{H}^{n_{i}}(B) \bigcap V \neq \varnothing
$$

We know that $B_{\varepsilon}(A)=U_{1}$ is an open set in $M$. According to topological transitivity for $U_{1}, V$ there exists compact set $A_{1}^{\prime} \subseteq U_{1}$ such that

$$
\mathrm{H}^{n}\left(A_{1}^{\prime}\right) \bigcap V \neq \varnothing
$$

We consider $\mathrm{H}^{n+1}\left(U_{1}\right)=U_{2} . B_{0}=A$ suppose that $B_{i}=B_{i-1} \cup A_{i}^{\prime}$, we can easily that $d_{H}\left(B_{i}, A\right)<\varepsilon$, $B=\bigcup_{i=1}^{\infty} A_{i}^{\prime} \cup A$ compact, then the set $B$ is set transitive.

Lemma 4.3. Consider $\mathrm{H}$ as topological transitive and $M$ as compact manifold. If $A^{\prime} \in E q(\varphi)$, then $O\left(A^{\prime}\right)$ is $\varepsilon$ dense.

Proof. We need to prove that for every $\varepsilon>0$ and $p \in M$ there exists $n \in \mathbf{Z}_{+}$that $\mathrm{H}^{n}\left(A^{\prime}\right) \bigcap B_{\varepsilon}(\{p\}) \neq \varnothing$. Since $A^{\prime} \in E q(\varphi)$ then there exists $\delta_{0}>0, d_{H}\left(A^{\prime}, B\right)<\delta_{0}$ then for every $n \in \mathbf{Z}_{+}, d_{H}\left(\mathrm{H}^{n}\left(A^{\prime}\right), \mathrm{H}^{n}(B)\right)<\frac{\varepsilon}{3}$. Since $\mathrm{H}$ is topological transitive for $U=B_{\delta_{0}}\left(A^{\prime}\right), V=B_{\frac{\varepsilon}{3}}(\{p\})$ then for every compact set $A^{\prime \prime} \subseteq B_{\delta_{0}}\left(A^{\prime}\right)$ such that $\mathrm{H}^{n}\left(A^{\prime \prime}\right) \bigcap B_{\frac{\varepsilon}{3}}(\{p\}) \neq \varnothing$. Consider $B^{\prime}=A^{\prime \prime} \bigcup A^{\prime}$, it is easy to see that $A^{\prime} \subset B_{\delta_{0}}\left(B^{\prime}\right)$ 
and $B^{\prime} \subseteq B_{\delta_{0}}\left(A^{\prime}\right)$ so

$d_{H}\left(A^{\prime}, B^{\prime}\right)<\delta_{0}$. So for every $n \in \mathbf{Z}_{+}$,

$$
\begin{gathered}
d_{H}\left(\mathrm{H}^{n}\left(A^{\prime}\right), \mathrm{H}^{n}\left(B^{\prime}\right)\right)<\frac{\varepsilon}{3}, \\
\mathrm{H}^{n}\left(A^{\prime \prime}\right) \bigcap B_{\frac{\varepsilon}{3}}(\{p\}) \neq \varnothing
\end{gathered}
$$

then there exists $x \in M$ that $d_{M}(x, p)<\frac{\varepsilon}{3}, x \in \mathrm{H}^{n}\left(A^{\prime \prime}\right)$. Since for every $n \in \mathbf{Z}_{+}$,

$$
d_{H}\left(\mathrm{H}^{n}\left(A^{\prime}\right), \mathrm{H}^{n}\left(A^{\prime} \cup A^{\prime \prime}\right)\right)<\frac{\varepsilon}{3}
$$

For every $n \in \mathbf{Z}_{+}$there exists $y_{n} \in A^{\prime}$ such that $d_{M}\left(\mathrm{H}^{n}\left(y_{n}\right), x\right)<\frac{\varepsilon}{3}$. Then

$$
d_{M}\left(p, \mathrm{H}^{n}\left(y_{n}\right)\right) \leq d_{M}(x, p)+d_{M}\left(x, \mathrm{H}^{n}\left(y_{n}\right)\right)<\frac{\varepsilon}{3}+\frac{\varepsilon}{3}<\varepsilon
$$

so $\mathrm{H}^{n}\left(y_{n}\right) \in B_{\varepsilon}(\{p\})$ then $\mathrm{H}^{n}\left(A^{\prime}\right) \bigcap B_{\varepsilon}(\{p\}) \neq \varnothing$.

Definition 4.4. 1) $\varphi$ is called minimal if for every $A \in \varphi, B \in \varphi$ and $\varepsilon>0, B \subseteq B_{\varepsilon}\left(\mathrm{H}^{n}(A)\right)$ is equivalent to $\overline{O_{H}(\{x\})}=M$, for every $x \in M$. In other words all members of $\varphi$ are set transitive i.e.

$\overline{O_{H}(A)}=\overline{\bigcup_{n \in \mathbf{Z}_{+}} H^{n}(A)}=M$.

2) A member $A \in \varphi$ is called minimal if subsystem $\overline{O_{H}(A)}$ is minimal.

3) A member $A \in \varphi$ is called almost periodic if subsystem $\overline{O_{H}(A)}$ is minimal and compact.

4) If the set of almost periodic sets is dense in $\varphi$, we say that $\left(\mathbf{Z}_{+}, \varphi\right)$ satisfies the

Bronstain condition. If in addition the subsystem $\left(\mathbf{Z}_{+}, \varphi\right)$ is topological transitive, we say that it is an

$M$-system.

5) A set $A \in \varphi$ is periodic if there exists $n \in \mathbf{Z}_{+}$such that $H^{n}(A)=A$. If $\mathrm{H}$ is a topological transitive and the set of periodic sets is dense, then we say that it is a $P$-system.

6) A subset $P \subseteq \mathbf{Z}_{+}$is (left) syndetic if there exists a finite set $F \subseteq \mathbf{Z}_{+}$such that

$-F P=\mathbf{Z}_{+}$.

Proposition 4.5. If $\varphi$ is compact with respect to Hausdroff metric $d_{H}$ then a member in $\varphi$ is minimal iff it is almost periodic.

Proof $(\leftarrow)$ : This result is trivial, because $A \in \varphi$ is almost periodic then $\overline{O_{H}(A)}$ is minimal and compact then $A \in \varphi$ is minimal.

$(\rightarrow): \overline{O_{H}(A)}$ is minimal holds, we need to show that $\overline{O_{H}(A)}$ is compact. We have 


$$
\overline{O_{H}(A)}=\overline{\left\{\mathrm{H}^{n}(A): A \in \varphi, n \in \mathbf{Z}_{+}\right\}}=\overline{\left\{\mathrm{H}(A), \mathrm{H}^{2}(A), \mathrm{H}^{3}(A), \ldots\right\}} .
$$

Because $A$ is compact, we use continuity of $\mathrm{H}$ and $\mathrm{H}^{n}(A)$ is compact.

Because for every $n \in \mathbf{Z}_{+},\left\{A, \mathrm{H}(A), \mathrm{H}^{2}(A), \mathrm{H}^{3}(A), \ldots\right\}$ is collection of sets in $\varphi$ then $\overline{O_{H}(A)}$ is closed collection of compact collection $\varphi$, every closed subset of compact set is compact.

\section{References}

1. A. S. Gorodetski, Yu. S. Ilyashenko, Certain Properties of Skew Products over a Horseshoe and a Solenoid, Proceedings of the Steklov Institute of Mathematics, Vol. 231, 2000, pp. 90-112.

2 B. Weiss, Topological transitivity and ergodic measures Math. Syst. 1971, Theory 5715.

3. D. Lind,B. Marcus, Symbolic Dynamics and Coding, 1995, Cambridge: Cambridge University Press.

4. E. Akin, S. Kolyada, Li-Yorke sensitivity, Institute of physics publishing, Nonlinearity 16 (2003) 1421-1433.

5. E. Glasner, B. Weiss, Sensitive dependence on initial conditions, Nonlinearity 6 (1993) 1067-1075.

6. E. Kontorovich, M. Megrelishvili, A note on sensitivity of semigroup actions, Semigroup Forum (2008) 76: 133-141.

7. E. Murinova, Generic chaos in metric spaces, Acta Univ. M. Belii. Math. no. 8(2000), pp.43-50.

8. J. Banks, J. Brooks, G. Cairns, G. Davis and P. Stacey, On Devaney's defnition of chaos, Am. Math. Mon. 99 (1992), 332-334.

9. K. T. Alligood, chaos, An introduction in dynamical systems, springer-verlag, 1996.

10. M. Brin, G. Stuck, Introduction to Dynamical Systems (Cambridge: Cambridge University Press), 2002.

11. R. Berglund, M. Vellekoop, On intervals, transitivity = chaos, Am. Mon. 101 353-5.

12. R. Clark, Dynamical Systema, Stability, Symbolic Dynamics, and Chaos, CRC press, 1995.

13. R. S. Li, A note on stronger forms of sensitivity for dynamical systems, Chaos, Solitons and Fractals 45 (2012), $753-$ 758.

14. S. Ruette, Chaos for continuous interval mapsa survey of relationship between the various sorts of chaos Bookpreprint available from www.math.u-psud.fr/ruette/publications.html.

15. S. Shao, X. Ye and R. Zhang, Sensitivity and regionally proximal relation in minimal systems, Science in chaina series A: Mathematics, Jan., 2008, Vol. 51, No. 6, 987-994.

16. T. K. S. Moothathu, Stronger forms of sensitivity for dynamical systems,2007 Nonlinearity 202115.

17. W. Huoyun, L. Xioongwu, F. Heman, Sensitivity and chaos of semi group actions, Springer, (2011), 81-90.

18. W. Huang, X. Ye, An explicit scattering, non-weakly mixing example and weak disjointness, Nonlinearity 15(2002) 84962. 